\title{
Mauss et Sigaut
}

Réflexions sur les liens entre les techniques et le genre

Mauss, Sigaut, techniques and gender

\section{Fabien Knittel et Pascal Raggi}

\section{(2) OpenEdition \\ Journals}

Édition électronique

URL : http://journals.openedition.org/artefact/3304

DOI : 10.4000/artefact.3304

ISSN : 2606-9245

Éditeur :

Association Artefact. Techniques histoire et sciences humaines, Presses universitaires du Midi

Édition imprimée

Date de publication : 15 mars 2019

Pagination : 215-235

ISBN : 978-2-8107-0623-5

ISSN : 2273-0753

\section{Référence électronique}

Fabien Knittel et Pascal Raggi, « Mauss et Sigaut », Artefact [En ligne], 9 | 2018, mis en ligne le 04 mars 2020, consulté le 29 novembre 2020. URL : http://journals.openedition.org/artefact/3304; DOI : https://doi.org/10.4000/artefact.3304

\section{cc) $(1) \Theta$}

Artefact, Techniques, histoire et sciences humaines est mise à disposition selon les termes de la Licence Creative Commons Attribution - Pas d'Utilisation Commerciale - Pas de Modification 4.0 International. 


\section{Mauss et Sigaut}

Réflexions sur les liens entre les techniques et le genre Fabien Knittel et Pascal Raggi

\section{Résumé}

Les liens entre genre et techniques ont suscité des questionnements concernant les rapports entre les femmes et les techniques. Les problématiques du genre invitent à dépasser cette histoire centrée sur un seul sexe et à poser des questions relatives à la co-construction du masculin et du féminin. Ces réflexions, à la croisée du genre et des techniques, méritent d'être approfondies. Dans cette perspective, nous avons fait le choix de réfléchir à l'apport des travaux de spécialistes des techniques, Marcel Mauss (1872-1950) et François Sigaut (1940-2012), même si ces deux auteurs n'ont pas écrit directement sur le genre ni utilisé le concept de genre, pour essayer de comprendre les interrelations entre genre et techniques. Nous pensons que leurs œuvres, relues à la lumière des auteur.es spécialistes des gender studies, aident à saisir la complexité des rapports entre genre et techniques. Mais pour cela il est nécessaire de faire " un pas de côté » théorique. Les réflexions de Mauss et de Sigaut deviennent alors des «boîtes à outils » pour l'étude des liens entre genre et techniques et l'on peut considérer que ces deux auteurs ont contribué à un redimensionnement de l'histoire des techniques pouvant inclure pleinement l'histoire du genre (et réciproquement), notamment en dépassant une séparation trop stricte entre l'étude de la conception et celle de l'usage des techniques.

\section{Mots-clés}

Marcel Mauss, François Sigaut, histoire des techniques, $x x^{e}$ siècle, genre

95 Fabien Knittel et Pascal Raggi, « Mauss et Sigaut. Réflexions sur les liens entre les techniques et le genre », Artefact, 9, 2018, p. 215-235. 


\section{Mauss, Sigaut, techniques and gender}

\section{Abstract}

The links between gender and techniques have caused some questions about the relationships between women and techniques. However, the key issues on gender tend to go beyond this history only focused on one sex and to ask questions relative to the coconstruction of the masculine and the feminine. At the cross-roads of gender and techniques, these thoughts deserve to be dug deeper. In this way, we have chosen to explore the works of two French specialists of techniques, Marcel Mauss (1872-1950) and François Sigaut (1940-2012) - although they did not write on gender nor used the gender concept - in order to try to understand the interrelations between gender and techniques. We think that their works, put in relation with those gender studies specialists, help to grasp the complexity of connections between gender and techniques. But, to do that, we need to take a theoretical step aside. Mauss and Sigaut's thoughts can then be considered as "tool boxes" for the study of the links between gender and techniques. These authors can be seen as having contributed to a reappraisal of the history of technology fully integrating gender history (and vice versa), especially by overstepping a too strict separation between the study of the creation and the study of the techniques' uses.

\section{Keywords}

Marcel Mauss, François Sigaut, history of technology, $20^{\text {th }}$ century, gender 


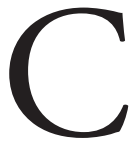

omme le mentionne le titre de l'ouvrage de Sylvie Schweitzer, les femmes ont toujours travaillé1. L'étude des femmes au travail a été un point fort de l'historiographie française des années 1970$1980^{2}$. En effet, féminin et masculin s'élaborent et se réélaborent mutuellement mais dans une configuration sociale où inégalités et rapports de pouvoir assignent à chacun son "rôle " social. Roland Pfefferkorn comme Danièle Kergoat relèvent la centralité de l'univers du travail dans les rapports sociaux de sexe $e^{3}$. Ce dernier concept, comme celui de genre, permet d'insister sur les rapports asymétriques entre hommes et femmes, la plupart du temps favorables aux hommes. Or, les techniques, leur manipulation, sont presque toujours renvoyées au masculin, vision dominante d'un ordre "sexuellement ordonné ${ }^{4}$ ». Ce stéréotype a la vie dure, mais ne correspond que partiellement à la réalité, une fois que l'on s'est efforcé de mener une analyse fine des rapports entre genre et techniques 5 .

Le croisement entre histoire du genre et histoire des techniques a été plus tardif et moins fréquent dans l'historiographie francophone, surtout si on la compare à l'historiographie anglo-saxonne où les relations entre genre et techniques ont été étudiées bien davantage et depuis plus longtemps ${ }^{6}$.

1. Sylvie SCHWEITZER, Les femmes ont toujours travaillé. Une histoire du travail des femmes aux XIX et $X X^{e}$ siècles, Paris, Odile Jacob, 2002.

2. Michelle Perrot, Les femmes ou les silences de l'histoire, Paris, Flammarion, 1998 ; Martine Cocaud, Dominique Gobineau, "Introduction ", Annales de Bretagne et des pays de l'Ouest, 114-3, 2007, "Travail, femmes et genre », p. 37-41.

3. Danièle Kergoat, Se battre disent-elles..., Paris, La Dispute, 2012, p. 101-110 et p. 213-223 ; Roland Pfefferkorn, Genre et rapports sociaux de sexe, Lausanne, éd. Page 2, 2012.

4. Pierre Bourdieu, La domination masculine, Paris, Seuil, 1998, p. 28-29 et p. 130-131.

5. Fabien Knittel, Pascal RagGi, "Des techniques au genre et retour ", in Fabien Knittel, Pascal RAGGI (dir.), Genre et techniques (XIX'-XXI siècles), Rennes, Presses universitaires de Rennes, 2013, p. 11-21 ; Fabien Knittel, Pascal Raggi, "Sciences, techniques et genre », in Alain Bihr, Roland Pfefferkorn (dir.), Dictionnaire des inégalités, Paris, Armand Colin, 2014, p. 365-366.

6. Par exemple : Ruth Schwartz Cowan, "The Industrial Revolution in the home: household technology and social change in the $20^{\text {th }}$ century", Technology and Culture, $\mathrm{n}^{\circ} 17,1976$, p. 1-24; Martha Trescott (dir.), Dynamos and Virgins Revisited: Women and Technological Change in History, Metuchen (New Jersey), Scarecrow Press, 1979. L'analyse féministe marxiste des techniques tant en histoire qu'en sociologie ou anthropologie a été d'un apport décisif : Cynthia CockBurn, "Women's access to European industrial relations", European Journal of Industrial Relations, juillet 1995, vol. 1-2, p. 171-189 ; Helen Harden Chenut, The Fabric of Gender: Working-Class Culture in Third Republic France, Pennsylvania State University Press, 2005 ; Danielle Chabaud-Rychter, Genre et techniques domestiques, Paris, L'Harmattan, 1998 et Paola TABET, La construction sociale de l'inégalité des sexes : des outils et des corps, Paris, L'Harmattan, 1998 entre autres. Approche renouvelée par un courant d'analyse féministe des techniques, voir Judy Wajcman et al. (dir.), The Social 
Il n'empêche, depuis la fin des années 1990 et le début des années 2000, des travaux francophones sont venus combler cette lacune et, depuis, les travaux se sont multipliés ${ }^{7}$.

Les liens entre genre et techniques ont suscité, dans un premier temps, des questionnements concernant les rapports entre les femmes et les techniques ${ }^{8}$. Or, les problématiques du genre invitent à dépasser cette histoire centrée sur un seul sexe et à poser toutes les questions relatives à la construction de la masculinité, y compris celles relatives à la virilité L'enseignement technique, par exemple, contribue à la construction d'un certain type de masculinité. Les différences genrées sont souvent accentuées à l'école ${ }^{10}$ et perpétuées, voire renforcées (et très rarement atténuées) au sein des milieux professionnels ${ }^{11}$.

Cependant, un certain nombre de questions à la croisée du genre et des techniques méritent encore d'être approfondies et, pour certaines, « remises sur le métier ", pour tenir compte des avancées de la recherche à la fois chez les spécialistes du genre et chez les " technologues », qui ignorent encore

Shaping of Technology, Philadelphia, Open University Press, 1999 [1985], p. 177-198 et "Gendered innovations in science, health and medicine, engineering and environment" : http://genderedinnovations.stanford.edu/.

7. Delphine Gardey, La dactylographe et l'expéditionnaire. Histoire des employés de bureau (18901930), Paris, Belin, 2001 ; D. Chabaud-Rychter, Genre et techniques domestiques, op. cit. ; Laurence TAIN, Le corps reproducteur, Rennes, Presses de l'EHESP, 2013.

8. Christine BARD, "L'impact du genre ", in Jean-François SirInelli et al. (dir.), Les historiens français à l'œuvre 1995-2010, Paris, PUF, 2010, p. 205-235.

9. Sonya O. Rose, What is Gender History?, Cambridge, Polity Press, 2012 ( $1^{\mathrm{re}}$ éd. 2010), p. 56-79; Alain Corbin, Jean-Jacques Courtine, Georges Vigarello (dir.), Histoire de la virilité, Paris, Seuil, 2011, 3 vol. ; Anne-Marie Sohn, "Sois un homme!" : la construction de la masculinité au XIX siècle, Paris, Seuil, 2009 ; Paul SмIтH (dir.), Boys: Masculinities in Contemporary Culture, Boulder, Westview Press, 1996 ; Judith Halberstam, "An Introduction to Female masculinity: masculinity without men", in Rachel Adams, David Savran (dir.), The Masculinity Reader, Oxford, Blackwell, 2002, p. 55-375.

10. Marie-Claire Dauvisis, Danielle Morali, Brigitte SiCard (dir.), Culture technique et formation, Nancy, Presses universitaires de Nancy, 1991 ; Sharif Gemie, Women and Schooling in France 1815-1914. Identity, Authority, Gender, Keele, Keele University Press, 1995 ; Marianne Thivend, "Former filles et garçons à un métier : jalons pour une histoire sexuée des formations techniques et professionnelles, $\mathrm{XIX}^{\mathrm{e}}$-Xx $\mathrm{x}^{\mathrm{e}}$ siècles ", in F. Knittel, P. RaGGi (dir.), Genre et techniques, op. cit., p. 25-37.

11. Elles peuvent même exister dans le cadre des grèves. À partir des années 1960-1970, des formes d'insubordination ouvrière fortement genrées s'expriment, tandis que le rôle des femmes dans les grèves a beaucoup évolué depuis les mouvements sociaux de la première moitié du $\mathrm{xx}^{\mathrm{e}}$ siècle. Voir Xavier Vigna, Histoire des ouvriers en France au XX' siècle, Paris, Perrin, 2012, p. 270-272. 
trop les travaux des un.es et des autres. C'est pourquoi nous avons fait le choix, ici, de réfléchir à l'apport des travaux de spécialistes des techniques, l'un au début du xx ${ }^{\mathrm{e}}$ siècle, Marcel Mauss (1872-1950) ${ }^{12}$, l'autre de la seconde moitié $\mathrm{du} \mathrm{xx}^{\mathrm{e}}$ et du début $\mathrm{du} \mathrm{XxI}^{\mathrm{e}}$ siècle, et qui est, en partie, héritier et continuateur de Mauss, François Sigaut (1940-2012) ${ }^{13}$, pour apporter notre contribution à la compréhension des relations entre genre et techniques. Toutefois, ces deux auteurs n'ont ni écrit directement sur le genre ni utilisé le concept de genre ou un autre s'en approchant et questionnant les rapports sociaux de sexes dans leurs travaux. Nous pensons que leurs œuvres respectives peuvent aider à saisir la complexité des rapports entre genre et techniques mais pour cela il est nécessaire de faire " un pas de côté " théorique. Les réflexions de Mauss et de Sigaut deviennent alors des «boîtes à outils » (au sens foucaldien de l'expression) pour l'étude des liens entre genre et techniques. Bien sûr, tous les textes de Mauss et Sigaut ne sont pas « utilisables » pour aborder cette question. Seule une partie de leur œuvre sera donc étudiée ici au risque, certes, de certains partis pris.

Nous voudrions principalement ouvrir des pistes de réflexion et proposer des perspectives d'ordre théorique. Nous cherchons à réfléchir à partir de la confrontation des œuvres multiples qui ont participé à faire comprendre les liens entre genre et techniques en mettant en avant les apports possibles des travaux de Mauss et Sigaut, repris à nouveaux frais dans cette perspective que, ni l'un ni l'autre, n'avaient envisagée ${ }^{14}$.

\section{De l'histoire des techniques au genre : (re)partir de Mauss et de ses héritiers}

À partir du XIX ${ }^{e}$ siècle, l'histoire des techniques a connu un essor important conjointement, et tout à la fois, à la progression de la vulgarisation des

12. Marcel Fournier, Marcel Mauss, Paris, Fayard, 1994 ; Jean-François Bert, L'atelier de Marcel Mauss, Paris, CNRS Éditions, 2012.

13. Il faut noter le site internet dédié à la mémoire et à la perpétuation de l'œuvre de F. Sigaut : http://www.francois-sigaut.com/. On y trouve les références de ses travaux ainsi que de nombreuses archives inédites.

14. Toutefois, développer longuement des exemples précis pour valider ou invalider ces propositions n'est pas notre objectif. Une application grâce à une ou plusieurs études de cas, menées à partir des cadres théoriques élaborés par Mauss et/ou Sigaut croisés avec les études de genre, demanderait la rédaction d'un nouvel article. 
connaissances techniques, à la volonté de la part de certains techniciens de mieux connaître leurs techniques, à l'intérêt pour la découverte de techniques anciennes et au désir d'expliquer les évolutions sociales en utilisant des éléments d'histoire des techniques ${ }^{15}$. En 1935, la revue les Annales consacra un numéro spécial à l'histoire des techniques pour encourager les historiens à renouveler un domaine historiographique structuré avant la Première Guerre mondiale dans lequel il demeurait (et reste encore aujourd'hui) beaucoup de thèmes à aborder. Au $\mathrm{xx}^{\mathrm{e}}$ siècle, le développement de la société de consommation dans le cadre de pratiques sociales et d'une vie quotidienne de plus en plus liées aux techniques (depuis l'utilisation du téléphone jusqu'à celle des réseaux informatiques et de l'Internet, en passant par l'évolution des façons de se déplacer sur terre, sur l'eau et dans les airs) a compliqué les recherches sur les techniques et leur histoire. Or, comme l'écrivait Lucien Febvre, «l'activité technique ne saurait s'isoler des autres activités humaines ${ }^{16}$ "; idée-force reprise par de nombreux technologues comme François Sigaut par exemple ${ }^{17}$. Dans la première moitié $\mathrm{du} \mathrm{Xx}^{\mathrm{e}}$ siècle, les liens entre genre et techniques sont encore peu abordés, mais les pistes ouvertes par Mauss concernant les techniques du corps méritent d'être rappelées tant elles nous semblent fécondes pour l'étude genrée des techniques.

Pour définir les techniques, Mauss propose un cadre assez large et bien connu : il appelle technique " un acte traditionnel efficace ». Mais ajoute aussitôt : "il faut qu'il soit traditionnel et efficace. Il n'y a pas de technique et pas de transmission, s'il n'y a pas de tradition. C'est en quoi l'homme se distingue avant tout des animaux : par la transmission de ses techniques... ${ }^{18}$. Ailleurs, il précise que les techniques sont " des actes traditionnels groupés en vue d'un effet mécanique, physique ou chimique ${ }^{19}$ ". Dans son œuvre, Marcel Mauss aborde peu la question du genre, et les rapports sociaux de sexe liés aux techniques restent une thématique à la marge de ses préoccupations alors que le rapport au corps et les techniques

15. Bertrand Gille, Histoire des techniques, Paris, Gallimard, coll. «La Pléiade », 1978, p. 5-6.

16. Lucien Febvre, "Réflexions sur l'histoire des techniques ", Annales d'histoire économique et sociale, $\mathrm{n}^{\circ} 36,1935$, p. 531-535, p. 532.

17. François Sigaut, Comment Homo devint faber, Paris, CNRS Éditions, 2012.

18. Marcel Mauss, "Les techniques du corps ", in Sociologie et anthropologie, Paris, PUF, 1935, rééd. "Quadrige ", 2009, p. 371 ; Marcel Mauss, Techniques, technologie et civilisation, Paris, PUF, "Quadrig", 2012, p. 365-394.

19. Marcel Mauss, Manuel d'ethnographie, Paris, Payot, 2002 ( $1^{\text {re }}$ éd. 1967), p. 49. 
corporelles sont au cœur de celles-ci. Néanmoins, dans l'article consacré aux techniques du corps, article aujourd'hui considéré comme fondateur des études sociohistoriques sur le corps ${ }^{20}$, la division entre les sexes n'est pas oubliée $^{21}$. Marie-Elisabeth Handman, dans son texte récent consacré à la place du genre dans l'œuvre de Mauss, indique que, si le concept n'est bien évidemment pas au cœur de l'œuvre maussienne, Mauss est loin d'être sexiste ou même indifférent et il apparaît comme un "ethnologue [...] attentif aux femmes ${ }^{22}$ ». Il n'y consacre pas de longs développements; en revanche, on trouve des notations éparses dans l'ensemble de son œuvre. Irène Théry montre quaujourd'hui les réflexions sur le genre peuvent gagner à utiliser les cadres de la pensée " technicienne » de Mauss : " la sociologie maussienne du symbolisme et de l'institution offre aux sciences sociales, avec la "division par sexes" ", un outil précieux, qui peut être fructueusement prolongé et retravaillé aujourd'hui ${ }^{23}$ ".

Maurice Halbwachs a précisé la définition maussienne des techniques. Pour lui, l'homme, c'est-à-dire l'être humain, se définit par sa technique et plus largement par la technique ; technique qui n'existe que par le corps. M. Halbwachs n'affirmait-il pas que "l'homme est un animal qui pense avec ses doigts " ${ }^{24}$ ? Cette définition des techniques est complétée par ce que François Sigaut nomme la "formule de Mauss ", c'est-à-dire la technique comprise comme une action finalisée et efficace ${ }^{25}$. Car l'acte technique

20. M. Mauss, «Les techniques du corps », op. cit. ; Olivier Martin, Dominique Memmi, « Marcel Mauss. La redécouverte tardive des Techniques du corps ", in Dominique Memmi et al. (dir.), La tentation du corps. Corporéité et sciences sociales, Paris, Éditions de l'EHESS, 2009, p. 23-46.

21. Marie-Elisabeth HandMAN, "Marcel Mauss et la division par sexes des sociétés : un programme inaccompli ", in Danièle Chabaud-Rychter et al. (dir.), Sous les sciences sociales, le genre, Paris, La Découverte, 2010, p. 52-63, p. 55. Précisons que l'article de Mauss est l'un des premiers cités dans l'introduction (p. 14) de l'Encyclopédie critique du genre parue en 2016 : Juliette Rennes et al., "Introduction. La chair des rapports sociaux ", in Juliette Rennes (dir.), Encyclopédie critique du genre, Paris, La Découverte, 2016, p. 13-31.

22. M.-E. Handman, « Marcel Mauss et la division par sexes des sociétés ", op. cit., p. 54.

23. Irène ThÉRY, "La notion de division par sexe chez Marcel Mauss ", L’Année sociologique, vol. 53-1, 2003, p. 33-54.

24. Cité par Raymond Tallis, The Hand: A philosophical Inquiry in Human Being, Edinburgh University Press, 2003, p. 205. Idée à relier au fameux aphorisme d'Anaxagore, cité par Aristote, qui assure que l'homme est intelligent parce qu'il a des mains. Kant affirme lui aussi que « la main est la fenêtre ouverte sur l'esprit ", cité par Richard Sennett, Ce que sait la main, Paris, Albin Michel, 2010, p. 205.

25. François Sigaut, "La formule de Mauss ", Techniques et culture, n 54-55, 2010 (première publication dans la même revue en 2003), p. 357-367 ; François Sigaut, « Retour sur "La Formule 
n'est pas seulement mécanique, il est aussi une pensée, un acte réfléchi : c'est la "main intelligente " évoquée par Richard Sennett ${ }^{26}$. Depuis l'Antiquité, d'Anaxagore à Aristote, puis, bien plus tard, avec Kant, nombreux sont ceux qui jugent que les êtres humains pensent parce qu'ils possèdent des mains et qu'ils sont en mesure de manipuler des objets ${ }^{27}$. De son côté, Matthew Crawford insiste sur l'importance de la manipulation dans le rapport matériel au monde ${ }^{28}$. André Leroi-Gourhan ajoute que "l'outil n'est réellement que dans le geste qui le rend techniquement efficace ${ }^{29}$. Les techniques sont donc entendues de façon très générale comme des actes à finalité efficace et non pas seulement comme l'usage d'outils ou de machines. Marcel Mauss a parfaitement montré que la marche ou la natation relèvent des techniques à proprement parler ${ }^{30}$.

Les techniques forment donc un ensemble qui inclut le corps, l'intellect et les outils/machines; tous sont utilisés dans l'acte technique. Faire l'histoire des techniques ${ }^{31}$ a ainsi permis de définir la notion de "système technique » : " en règle générale, toutes les techniques sont, à des degrés divers, dépendantes les unes des autres, et il faut nécessairement entre elles une certaine cohérence : cet ensemble de cohérences aux différents niveaux de toutes les structures de tous les ensembles et de toutes les filières compose

de Mauss" ", Techniques et culture, $\mathrm{n}^{\circ}$ 54-55, 2010, p. 354-356.

26. R. Sennett, Ce que sait la main, op. cit., p. 237-239.

27. Nous l'avons déjà précisé et analysé dans F. Knittel, P. RAGGI, "Des techniques au genre et retour ", op. cit., p. 15.

28. Matthew Crawford, Éloge du carburateur, Paris, La Découverte, 2010.

29. Cité par Antoine Casanova, "Préface ", in Charles Parain, Outils, ethnies et développement historique, Paris, Éditions sociales, 1979, p. 11.

30. M. Mauss, "Les techniques du corps ", op. cit..

31. De grandes œuvres historiques ont abordé le thème de l'évolution générale des techniques des origines au temps présent comme, en anglais, l'ouvrage $A$ History of Technology, Oxford, Clarendon Press, London, Oxford University Press, 1954-1958, 5 vol., et, en français, Maurice Daumas (dir.), Histoire générale des techniques, Paris, PUF, 1962-1968, 3 vol. Depuis la fin du xxe siècle, et alors que le livre de Bertrand Gille, Histoire des techniques tend encore vers l'exhaustivité, la complexification des études sur ce thème oriente les recherches vers des choix de présentation non-exhaustive de l'histoire de certaines techniques ; voir, par exemple, Bruno JаСомY, Une histoire des techniques, Paris, Seuil, 1990. Aujourd'hui, la revue britannique History of Technology apparait comme une publication qui fait la promotion d'études renouvelant les thèmes d'histoire des techniques. Les précisons et nuances apportées par David Edgerton sont fondamentales aussi, principalement l'idée que les innovations techniques ne remplacent pas purement et simplement les anciennes techniques et qu'il y a coexistence de l'ancien et du nouveau/moderne : David EDgerton, Quoi de neuf? Du rôle des techniques dans l'histoire globale, Paris, Seuil, 2013 (1 $1^{\mathrm{re}}$ éd. anglaise, 2006). 
ce que l'on peut appeler un système technique ${ }^{32}$ ». Le fait technique dépend aussi, si l'on suit Leroi-Gourhan, des conditions du milieu ${ }^{33}$. Les systèmes techniques possèdent une dimension sociale :

Les techniques matérielles - ou encore les objets - ne cessent de recomposer le social, la considération des techniques intellectuelles et symboliques, algorithmiques et gestionnaires - en un mot peut-être maladroit, la considération des techniques non matérielles - contribue plus encore à mettre en péril les représentations paresseuses du social dans la mesure où la relation humaine, dans toutes ses dimensions (culturelles et sociales, techniques et politiques, scientifiques et managériales), est la cible même de leur intervention. Le technique est social, mais le social est aussi technique ${ }^{34}$.

Le cadre d'analyse le plus pertinent est donc devenu celui des systèmes sociotechniques dont Madeleine Akrich a expliqué la structuration et le fonctionnement ${ }^{35}$.

Aujourd'hui, à la lecture de l'historiographie des trente dernières années, il est possible d'affirmer que le genre contribue aussi à la définition du fait technique et qu'il est un des éléments structurants des systèmes sociotechniques. Dans le cadre des rapports entre genre et techniques, il faut interroger la place des femmes, et leurs rapports avec les hommes, dans ces mêmes systèmes techniques à l'intérieur desquels des différences genrées subsistent ${ }^{36}$. En effet, l'humain et le social mettent en forme et en œuvre les objets techniques : "Lors des multiples interactions avec la matière qui est

32. B. Gille, Histoire des techniques, op. cit., p. 19. Sur les limites d'une explication des évolutions du travail humain par les seuls changements de système technique : Yves Schwartz, Expérience et connaissance du travail, Paris, Messidor/Éditions sociales, 1988, p. 407-437.

33. André Leroi-Gourhan, Évolution et techniques. L'homme et la matière, Paris, Albin Michel, 1971 ( $1^{\text {re }}$ éd. 1943), et Milieu et techniques, Paris, Albin Michel, 1973 ( $1^{\text {re }}$ éd. 1945).

34. Yves Cohen, Dominique Pestre, «Présentation », «Histoire des techniques », Annales. Histoire, Sciences Sociales, 53 année, $\mathrm{n}^{\circ}$ 4-5, 1998, p. 721-744, p. 739.

35. Madeleine AKRICH, « La construction d'un système socio-technique. Esquisse pour une anthropologie des techniques ", Anthropologie et sociétés, n 13, 1989, p. 31-54.

36. L. TAIN, Le corps reproducteur, op. cit.; Delphine GARDEY, Ilana LöWY (dir.), Linvention du naturel. Les sciences et la fabrication du féminin et du masculin, Paris, Éditions des archives contemporaines, 2000 ; Nelly Oudshoorn, Beyond the Natural Body, an archeology of sex hormones, Londres/ New York, Routledge, 1994 et "Hormones, techniques et corps. L’archéologie des hormones sexuelles (1923-1940)", Annales Histoire Sciences Sociales, juillet-octobre 1998, nº 4-5, p. 775-793. 
le lot de tout acte technique quelque chose est certes opérationnalisé mais il est indissociablement pris, et à jamais, dans des jugements et des valeurs qui sont d'une autre nature ${ }^{37}$. " Précisément, ces jugements et ces valeurs ne peuvent pas forcément se défaire des conceptions genrées qui ont cours dans la société qui voit apparaître ou s'améliorer les objets techniques. Par exemple, dans le meilleur des cas, des ingénieurs ou des ingénieures peuvent concevoir des objets exempts de toute dimension genrée et, a contrario, il leur est possible, notamment dans le cadre de l'économie libérale de marché et sous la contrainte forte d'un marketing puissant, de fabriquer des objets genrés en jouant sur les prétendus goûts ou vertus féminines ou masculines pour les vendre. Par exemple, le marché des automobiles et son ciblage marketing sont, à cet égard, parfois caricaturaux : aux femmes les petites voitures pratiques et aux hommes les grosses berlines puissantes ${ }^{38}$.

Sous l'angle des enjeux de pouvoir ${ }^{39}$, les hommes ont intérêt à maintenir les dispositifs sociaux qui éloignent les femmes de l'univers des techniques. Cela contribue au pouvoir de domination masculin et le renforce ${ }^{40}$. Les techniques sont sources de pouvoir social et politique ${ }^{41}$. Paola Tabet a montré l'existence d'une " appropriation inégale des outils et des machines par les hommes et par les femmes dans la plupart des sociétés humaines ${ }^{42}$ ». Comme l'a écrit Michelle Perrot :

La place des femmes n'est pas réglée par la technique, mais par des questions de statut qui, traditionnellement, attribuent aux hommes les postes de commandement, d'encadrement, les outils compliqués, et aux femmes les tâches d'auxiliaires, d'aides, les travaux d'exécution, effectués à mains nues, peu spécialisés, voire casuels, et toujours subordonnés ${ }^{43}$.

37. Y. Cohen, D. Pestre, «Présentation », op. cit., p. 730.

38. Sur les différences de genre dans les comportements d'achat d'automobiles et leurs évolutions, voir notamment Robin WIDGERY, Jack MACGAUGH, "Vehicle message appeals and the new generation woman", Journal of Advertising Research, 33 (5), 1993, p. 36-42.

39. Joan W. Sсотт, "Le genre : une catégorie utile d'analyse historique ", in Joan W. Sсотт, De

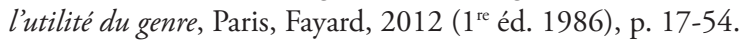

40. P. Bourdieu, La domination masculine, op. cit.

41. Danièle Chabaud-Rychter, Delphine Gardey (dir.), L'engendrement des choses. Des hommes, des femmes et des techniques, Paris, Éditions des archives contemporaines, 2002, p. 21.

42. Ibid., p. 22 ; Paola ТАВET, La construction sociale de l'inégalité des sexes : des outils et des corps, Paris, L'Harmattan, 1998.

43. M. Perrot, Les femmes ou les silences de l'histoire, op. cit., p. 181-182. 
Sans cesse les femmes sont renvoyées à la nature alors que les hommes dominent sciences et techniques, soit le domaine de la culture ${ }^{44}$. Il a déjà été montré par ailleurs que la neutralité des techniques était une illusion ${ }^{45}$. Les techniques et leurs usages induisent une conception du monde où les rapports masculin-féminin et l'identité genrée se structurent et se renouvellent au sein de réseaux sociotechniques ${ }^{46}$. Les techniques sont parties intégrantes de l'" environnement " social, économique, politique, culturel dont le genre est, en partie, constitutif. La construction de l'identité sexuée passe par une série d'interactions entre les sphères du langage, de l'école, de la famille, celles des objets et des techniques et bien d'autres encore ${ }^{47}$. Le sujet des rapports entre genre et techniques a donc déjà été abordé à maintes reprises mais les angles d'approche peuvent être variés et des relectures sont possibles. C'est le sens de nos réflexions sur ce que sont susceptibles de nous apporter les outils théoriques de Mauss, notamment ses propositions concernant les techniques du corps ${ }^{48}$.

Les techniques, comme le rapport aux techniques, sont construits socialement à l'instar des identités sexuées. De la même façon, le corps est un produit social ${ }^{49}$. À la suite des auteures de L'Engendrement des choses, il faut poser une interrogation essentielle pour comprendre l'interaction entre les sociétés et les techniques, sur les processus de coconstruction des techniques par le genre et du genre par les techniques : ce qui correspond à la notion d'engendrement proposée par Delphine Gardey et Danièle Chabaud-Rychter dans leur introduction : "les identités sexuées sont à proprement parler "engendrées" dans cette dynamique qu'est la construction individuelle de la personne de l'enfance à l'âge adulte [...] Du côté des techniques [...] il est alors question de comprendre en quoi [elles] ordonnent aussi le monde social et notamment les relations de genre ${ }^{50}$ ". Mais cela pose la question des sources disponibles pour écrire cette histoire

44. Philippe Descola, Par-delà nature et culture, Paris, Gallimard, 2005 ; Donna Harraway, Simians, Cyborgs, and Women: The Reinvention of Nature, Londres, Free Association Books, 1988.

45. D. Chabaud-Rychter, D. Gardey (dir.), L'engendrement des choses, op. cit., p. 48.

46. M. Akrich, "La construction d'un système socio-technique ", op. cit.

47. D. Chabaud-Rychter, D. Gardey (dir.), L'engendrement des choses op. cit., p. 90.

48. M. Mauss, "Les techniques du corps ", op. cit.

49. D. Chabaud-Rychter, D. Gardey (dir.), L'engendrement des choses..., op. cit., p. 21 ; Laurence Guignard, Pascal Raggi, Étienne Thévenin (dir.), Corps et machines à l'âge industriel, Rennes, Presses universitaires de Rennes, 2011.

50. D. Chabaud-Rychter, D. Gardey (dir.), L’engendrement des choses..., op. cit., p. 12. 
genrée des techniques. Les techniques du corps, pensées et analysées par Mauss, entrent donc dans cette construction sociale de l'identité de genre en même temps que celle du sexe $e^{51}$. La formation et le travail des ouvriers tullistes d'un côté, l'apprentissage familial et l'activité des dentellières de l'autre, sont un exemple d' "engendrement" des techniques ${ }^{52}$. Le tulle est fabriqué à l'usine de manière mécanique par des hommes tandis que la production dentellière à la main est effectuée par des femmes et jeunes filles au sein de l'espace domestique : "le développement du travail masculin du tulle mécanique rend visible l'identité sexuée du travail des dentellières ${ }^{53}$ ". Cet exemple d' " engendrement " montre l'importance des techniques du corps, au sens de Mauss, dans le processus même de l'assignation des individus à un genre particulier, en même temps qu'il met en lumière le processus inégalitaire de cette assignation. Comme Marcel Mauss, François Sigaut n'aborde pas directement les liens entre genre et techniques. Toutefois, son dernier ouvrage, Comment Homo devint faber ${ }^{54}$, permet d'élaborer des « outils " pour la réflexion sur cette thématique.

\section{Penser le genre des techniques avec Sigaut?}

Les réflexions de F. Sigaut sont stimulantes pour qui se propose de réfléchir aux implications de la "fabrique " technique du genre et de la construction genrée des techniques. L'étude et la compréhension des gestes techniques, qualifiés comme des "fonctions élémentaires de l'espèce humaine ${ }^{55}$ ", sont au cœur du travail de F. Sigaut et l'on sait par ailleurs que les gestes techniques sont, en partie, influencés par le genre de celui ou celle qui les exécute $^{56}$. Si pour l'espèce humaine " toutes les actions techniques sont outillées », « même en l'absence d'outil » précise Sigaut ${ }^{57}$, alors le genre des

51. Thomas Laqueur, La fabrique du sexe. Essai sur le corps et le genre en occident, Paris, Gallimard, 1992 ( $1^{\text {re }}$ éd. anglaise 1990).

52. Stéphane Lembré, « La qualification, la main et la machine. Filles et garçons face aux formations dentellières, $\mathrm{XIX}^{\mathrm{e}}$-Xx $\mathrm{x}^{\mathrm{e}}$ siècles ", in Fabien KnitTel, Pascal RaGGI (dir.), Genre et techniques..., op. cit., p. 53-68.

53. Ibid., p. 54.

54. F. Sigaut, Comment Homo devint faber, op. cit.

55. Ibid., p. 7.

56. Par exemple : S. Lembré, "La qualification, la main et la machine », op. cit.

57. F. Sigaut, Comment Homo devint faber, op. cit., p. 7 et p. 99. 
outils et des gestes outillés entre dans l'étude des influences réciproques entre genre et techniques. Pour Sigaut, c'est le corps qui agit comme un outil $^{58}$. Or, la question qui immédiatement se pose est de savoir si corps masculin et corps féminin sont "outillés " de la même manière et dans quelle mesure la naturalisation des comportements humains intervient dans une différenciation des " outillages " respectifs des corps sexués ? La réponse n'est qu'en partie connue. La manière dont se structure cette " action outillée ${ }^{59}$ " genrée du corps et dont elle évolue est, en fait, assez peu explorée et mérite des approfondissements importants. Mais il s'agit d'une recherche ambitieuse, compliquée à mener à bien tant les limites et les obstacles sont importants; l'un d'entre eux, et non des moindres, est celui des sources, cruciales pour l'historien -ne, qui s'avèrent le plus souvent " muettes » sur ce point. L'imprécision du vocabulaire en matière d'étude des techniques ne facilite en rien la tâche ${ }^{60}$.

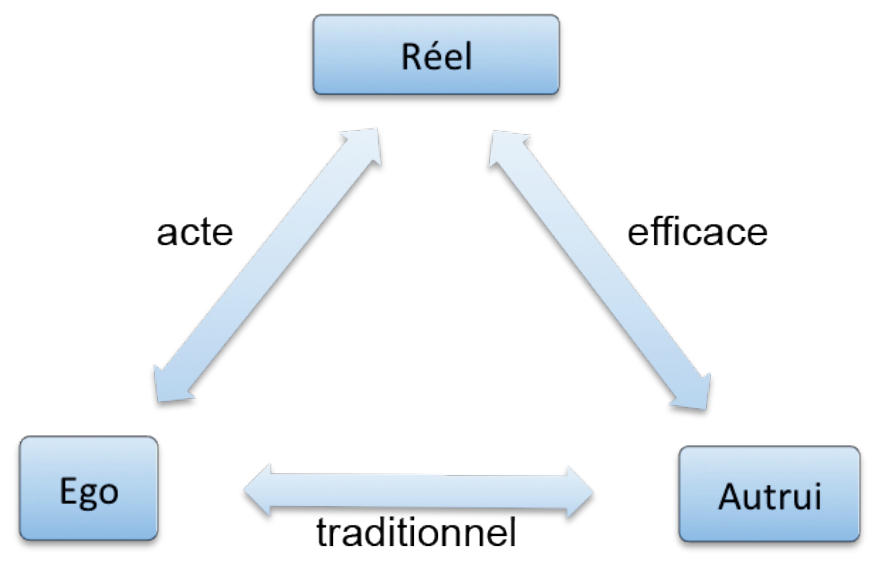

\section{Fig. 1. - Le « triangle » de Sigaut}

D’après François Sigaut, « Folie, réel et technologie. À propos de Philippe Bernardet, Les Dossiers noirs de l'internement psychiatrique, Paris, Fayard, 1989 ", Techniques \& Culture, $\mathrm{n}^{\circ} 15,1991$, p. 167-179.

Pour Sigaut, partant de l'œuvre de Mauss puis de celle de Bergson (non sans avoir souligné au préalable tout ce qui sépare ces deux penseurs), "l'outil suppose l'intelligence ", c'est-à-dire qu'un être humain

58. Ibid., p. 8 et p. 99.

59. Ibid., p. 15.

60. Ibid., p. 9. 
qui manipule accède à l'intelligence, c'est l'Homo Faber ${ }^{61}$. Il poursuit et indique que "l'intelligence est née par et pour la manipulation des choses matérielles ${ }^{62}$ ». Remarque d'autant plus intéressante si on ajoute la dimension genrée à l'étude des techniques. Ces aspects genrés des techniques trouvent leur place dans le "triangle de Sigaut ${ }^{63}$ " (Fig. 1), c'est-à-dire le schéma explicatif élaboré par Sigaut pour expliquer le rapport au monde, au réel ; le réel étant compris ici par Sigaut comme tout ce qui est matériel, c'est-à-dire l'ensemble des choses inanimées.

Sigaut montre que le rapport à autrui et au réel passe par les objets, donc les techniques. Pour le démontrer il superpose à son schéma ego-réel-autrui, la définition des techniques de Mauss, " un acte traditionnel et efficace ", pour réaliser un schéma heuristique, cadre possible d'explication du monde social. Plus tard il précise que « la configuration sociale élémentaire [...] est [...] un rapport ternaire mettant en jeu au moins deux personnes et un objet. La société humaine se construit comme humaine par le sens qu'elle donne aux objets matériels, car la relation hommes-objets est aussi nécessaire que la relation hommes-hommes pour la construction du sens ${ }^{64}$ ". Le genre des techniques, dans ce cadre explicatif, peut être intégré dans les rapports qu'ego entretient avec le reste de la structure sociale, c'est-à-dire le réel (le monde matériel, ce que Bruno Latour qualifie de non-humains ${ }^{65}$ ) et autrui, et représente un élément d'explication de son rapport d'être au monde. Quid des inégalités liées au sexe d'ego dans ce schéma explicatif ?

On sait que la domination masculine et les inégalités entre hommes et femmes sont à leur paroxysme dans la sphère professionnelle. L'étude des rapports sociaux de sexe au travail a permis de renouveler cette analyse des inégalités liées au genre dans la sphère professionnelle ${ }^{66}$. Pour les

61. Ibid., p. 19 et p. 134 .

62. Ibid., p. 21.

63. F. Sigaut, « Folie, réel et technologie. À propos de Philippe Bernardet, Les Dossiers noirs de l'internement psychiatrique, Paris, Fayard, 1989 ", Techniques \& Culture, n 15, 1991, p. 167-179. Il ne l'appelle pas lui-même comme cela. Ce sont les commentateurs et ceux qui l'utilisent comme cadre de réflexion théorique qui le désignent ainsi. Sigaut le nomme "triangle du sens " dans un article postérieur de 1992 : François Sigaut, "Le triangle du sens ", Techniques et culture, n 19 , 1992, p. 201-209, p. 205.

64. F. Sigaut, « Le triangle du sens », art. cit., p. 202.

65. Bruno Latour, Nous n'avons jamais été modernes. Essai d'anthropologie symétrique, Paris, La Découverte, 1997(1 $1^{\text {re }}$ éd. 1991).

66. H. H. Chenut, The Fabric of Gender, op. cit. 
historien·ne.s et les sociologues, l'étude du travail féminin ou des femmes au travail est toujours une problématique féconde ${ }^{67}$. C'est souvent l'occasion d'aller contre les stéréotypes des femmes cantonnées à l'espace domestique en montrant que l'espace public à travers le travail est aussi féminin, nonobstant la domination masculine. Le rapport que Dominique Poggi remet dès 1983 au ministre de la Culture de l'époque, insiste bien sur ce point et montre la difficulté pour les jeunes femmes à s'affirmer dans un monde du travail saturé de masculinitét ${ }^{68}$.

Nous avons là un cadre de réflexion pour saisir au plus près les stéréotypes de genre qui assignent artificiellement les hommes à la sphère des techniques et qui en écartent les femmes au prétexte d'aptitudes naturalisées différenciées selon le sexe ${ }^{69}$. Or les techniques domestiques sont en grande partie du ressort des femmes ${ }^{70}$, mais elles sont socialement dévalorisées. Par exemple, Frédérique El Amrani a montré que dans la première moitié $\mathrm{du} \mathrm{Xx}^{\mathrm{e}}$ siècle, les femmes ayant des savoir-faire professionnels acquis en travaillant dans des exploitations agricoles ont beaucoup de difficultés à les concevoir comme étant les composantes d'un véritable métier voire d'un statut professionnel ${ }^{71}$. Il faut attendre les années 1970-1980 pour que soit reconnue aux femmes d'agriculteurs la professionnalisation de leur travail, enfin considéré comme un vrai métier ${ }^{72}$. Précisément, c'est alors que le monde rural français se transforme radicalement, que le statut d'exploitante agricole - d'agricultrice - permet de reconnaître à leur juste valeur les

67. D. Kergoat, Se battre disent-elles..., op. cit.

68. Dominique Poggi, Les femmes dans la culture technique et scientifique : de l'oppression à l'innovation, Rapport du ministère de la Culture, 1983, dactylographié (66p.).

69. Delphine Gardey, Ilana Löwy, "Pour en finir avec la nature ", in D. Gardey, I. Löwy (dir.), L'invention du naturel, op. cit., p. 9-28; sur la théorie des «sphères séparées » et sur ses critiques, voir Catherine Hall, White, Male and Middle-Class: Explorations in Feminism and History, Cambridge, Polity Press, 1992, p. 94-108 ; Stéphanie Guyot-Knittel, Rebecca and Jane. Middle-Class Women as Source and Revelation of Class and Gender Tensions in two Victorian Novels, mémoire de master 2, Université de Lorraine, 2013, dactylographié, p. 56.

70. Entre autres, Danièle Chabaud-Rychter, "Women users in the design process of a food robot: Innovation in a French domestic appliance company", in Cynthia CocKBURN, Ruza FürsT-Dilic (dir.), Bringing Technology Home: Gender and Technology in a Changing Europe, Milton Keynes, Open University Press, 1994 ; D. Chabaud-Rychter, Genre et techniques domestiques, op. cit. 71. Frédérique El Amrani, "Femmes au travail dans les campagnes angevines durant le premier $\mathrm{xx}^{\mathrm{e}}$ siècle : quels mots pour quels travaux ?", Annales de Bretagne et des Pays de l'Ouest, tome 114, $\mathrm{n}^{\circ} 3$, 2007, p. 109-124.

72. Ibid., p. 123. 
tâches agricoles effectuées par les femmes ${ }^{73}$. On passe du travail au métier à part entière (qui sous-entend formation spécifique et reconnaissance professionnelle par les pairs).

Dans Comment Homo devint faber, Sigaut formule une hypothèse pour essayer de comprendre la division sexuelle des tâches et des techniques ${ }^{74}$. Il affirme que "dans l'espèce humaine $[. .$.$] ce sont les deux sexes qui se spé-$ cialisent l'un vis-à-vis de l'autre », avant d'expliquer que «la répartition des activités entre les sexes est la seconde des innovations fondamentales qui ont donné naissance à l'espèce humaine - la première étant le partage de l'attention ${ }^{75}$ ". Aujourd'hui la répartition des tâches entre les sexes perdure même si, Sigaut le remarque, "elle est de plus en plus contestée ${ }^{76}$ ". Cette répartition, inégalitaire, est liée aux mécanismes d'apprentissage séparés entre garçons et filles qui " doivent acquérir les savoir-faire propres à leur $\operatorname{sexe}^{77}$ ». Or cette idée de savoir-faire différenciés (que Sigaut appelle hétéropraxie) allant de soi apparaît comme une affirmation un peu rapide : ces tâches différenciées ne sont-elles pas déjà un construit social à la base des inégalités entre les sexes, le plus souvent naturalisées pour paraître allant de soi ? Cependant l'hypothèse de Sigaut est paléoanthropologique et il cherche l'origine de la séparation des tâches entre les sexes. Il propose alors de considérer "le plaisir de la réussite ", émulation à la base d'une séduction masculine des femmes, comme facteur explicatif de la différenciation des aptitudes techniques entre les femmes et les hommes. Sauf que le rôle de cet esprit agonistique à des fins de différenciations sexuelles dans le rapport aux techniques est très difficile à prouver ainsi que Sigaut l'admet lui-même à la fin de sa démonstration ${ }^{78}$. L'intérêt de cette réflexion, qui ne nous convainc pas totalement, est de proposer des hypothèses d'explication de phénomènes qui sont considérés comme évidents alors même que le rapport genré aux techniques est devenu, à l'ère historique, une construction sociotechnique.

Dans le monde professionnel, l'évolution technique permettant de soulager le travail à des postes particulièrement pénibles ne bénéficie pas

73. Ibid.

74. F. Sigaut, Comment Homo devint faber, op. cit., p. 173-184.

75. Ibid., p. 177-178.

76. Ibid., p. 180.

77. Ibid., p. 181.

78. Ibid., p. 182. 
forcément aux femmes auxquelles on peut persister à assigner certaines techniques jugées moins nobles que les techniques dont se prévalent les hommes ${ }^{79}$. Mais "l'action outillée ", pour genrée qu'elle soit, reste l'élément-clé de l'acte technique qui dépasse finalement, en partie, le clivage de genre. Ces réflexions stimulantes de F. Sigaut méritent donc d'être creusées dans le domaine de l'histoire des techniques en général et dans celui des rapports entre genre et techniques en particulier. Les techniques du corps, au sens de Mauss, ont été parfois marginalisées par les sciences humaines ${ }^{80}$, alors même que le corps lui-même est devenu, ces dernières années, un objet d'étude central pour ces mêmes sciences humaines ${ }^{81}$. Pour autant, et au-delà du paradoxe, ces études sur le corps en sciences humaines n'ont rien d'anecdotique et sont un révélateur des rapports inégalitaires de genre dans les sociétés humaines. Les relations de l'être humain aux techniques passent par le corps, mais un corps sexué bien sûr. Dans une approche naturaliste, ce corps devient limitant, notamment pour les femmes. L'histoire des liens entre genre et techniques est souvent une histoire de l'exclusion des femmes de la sphère des techniques où les hommes s'imposeraient du fait de leur dextérité et de leur force physique, ce que Cynthia Cockburn appelle le "pouvoir physique ${ }^{82}$. Aujourd'hui l'importance de la force physique des hommes, prétendue supérieure à celle des femmes, comme élément de la domination masculine est questionnée voire invalidée dans sa réalité anthropologique ${ }^{83}$.

79. Michelle Perrot, Les femmes ou les silences de l'histoire, Paris, Flammarion, 1998, p. 181-182.

80. F. Sigaut, «Le triangle du sens », art. cit., p. 36.

81. Par exemple : Bernard Andrieu, "Philosophie du corps ", Philosophie du corps, Expériences, interactions et écologie corporelle, Paris, Vrin, 2010, p. 7-59 ; L. GuIGnARD et al., Corps et machines à l'àge industriel, op. cit.

82. Cynthia Cockburn, “The Material of Male Power", in Donald MacKenzie, Judith Wajcman (dir.), The Social Shaping of Technology, Philadelphia, Open University Press, 1999, p. 17-198.

83. Priscille Touraille, Hommes grands, femmes petites: une évolution coûteuse. Les régimes de genre comme force sélective de l'évolution biologique, Paris, Éditions MSH, 2008. Dans cet ouvrage, l'auteure revient sur la pertinence des explications d'anthropologie biologique relatives aux différences de tailles entre les hommes et les femmes. Par exemple, elle se demande si les statures moins élevées des femmes sont le résultat d'une adaptation à des conditions nutritionnelles défavorables (p. 203227) ou si les femmes n'ont pas plutôt été victimes de privations nutritionnelles. La faible stature féminine n'étant alors pas un avantage pour la procréation mais plutôt une adaptation à un manque de nourriture : «Les conséquences de l'alimentation sur les femmes se matérialisent en des jeux de sélection et de contre-sélection sur leur stature. Les coûts obstétriques sont payés par les plus petites femmes dont l'organisme serait plus "adapté" à de mauvaises conditions nutritionnelles. Les coûts nutritionnels sont payés par les femmes de grande taille qui auraient par leur stature la caractéristique adaptative nécessaire pour surmonter les problèmes obstétriques » (p. 299). 
Cependant, les représentations restent encore fortes qui associent force physique et domination masculine, d'où l'intérêt de la notion de " pouvoir physique " de Cockburn qui permet de les mettre en question. Ces rapports sexués aux techniques se structurent durant l'enfance dans le cadre éducatif, principalement familial. Ils élaborent la différenciation sexuelle, en particulier à travers jeux et jouets ${ }^{84}$. Renvoyer le sexe à la nature et à la biologie n'est pas aussi simple car le sexe biologique est aussi soumis à une construction sociale où les techniques jouent d'ailleurs un rôle non négligeable ${ }^{85}$. Sexe et genre sont encore des notions qui doivent être abordées avec un sens critique fort car les évidences égarent le chercheur et lui masquent certaines réalités sociales incorporées difficilement objectivables ${ }^{86}$. Les rapports entre genre et techniques en font partie et renvoient de manière fréquente au corps. Cela est déjà connu mais il reste encore beaucoup à faire pour connaître plus précisément l'anthropologie et l'histoire des gestes techniques ${ }^{87}$ : quelles postures, quels gestes, adéquats ou maladroits?

L'action technique est "définie par l'évidence de ses effets matériels ${ }^{88}$ ", mais il existe une grande "part de non-dits dans l'action technique ${ }^{89}$ ». Ces non-dits jouent un rôle important dans l'assignation genrée des techniques et dans les constructions hiérarchiques entre techniques soi-disant féminines et techniques soi-disant masculines, favorisant les phénomènes de domination de tous ordres. À partir de la seconde moitié du XIx siècle, la construction de la virilité est une initiation (à l'école mais aussi à la caserne lors de la conscription à partir de la fin du XIX siècle) à l'obéissance ainsi qu’à la résistance physique et morale à la violence ${ }^{90}$. La virilité exige la

84. Michel Manson, «Les jouets et la différenciation sexuelle, une longue histoire culturelle ", in D. Chabaud-Rychter, D. Gardey (dir.), L'engendrement des choses..., op. cit., p. 103-122, p. 103. 85. D. Gardey, I. Löwy (dir.), L'invention du naturel..., op. cit., p. 9-28.

86. T. Laqueur, La fabrique du sexe, op. cit.

87. Didier Bouillon, André Guillerme, Martine Mille, Gersende Piernas (dir.), Gestes techniques, techniques du geste, Villeneuve-d'Ascq, Presses universitaires du Septentrion, 2017.

88. F. Sigaut, «Le triangle du sens ", art. cit., p. 64.

89. Ibid., p. 66.

90. André Rauch, Crise de l'identité masculine, 1789-1914, Paris, Hachette, 2000, p. 177-210. Voir aussi Alain Corbin, Georges Vigarello, Jean-Jacques Courtine (dir.), Histoire de la virilité, t. 2 : Le triomphe de la virilité, le XIXe siècle, Paris, Seuil, 2011 et Odile Roynette, Bon pour le service. L'expérience de la caserne en France à la fin du XIXe siècle, Paris, Belin, 2000. Enfin : Odile RoynetTE, "La construction du masculin. De la fin du XIX siècle aux années 1930 ", Vingtième siècle, n ${ }^{\circ} 75$, 2002-3, p. 85-96. 
domination de soi et la maîtrise des affects. Sous la III ${ }^{e}$ République, l'instruction scolaire diffuse une culture nouvelle, celle de la citoyenneté dans un contexte d'expansion technique et scientifique ${ }^{91}$. L'école républicaine forme des individus qualifiés tout en assurant l'éducation et la socialisation des futurs citoyens ${ }^{92}$. Le but est dorénavant "de faire intérioriser à l'élève le sens du devoir ${ }^{93}$ ", c'est-à-dire d'inculquer aux jeunes garçons l'habitus du citoyen. C'est dans ce but que l'institution scolaire met en œuvre un ensemble de dispositifs de contrôle et de surveillance qui vise à la normalisation des comportements ${ }^{94}$; normes le plus souvent genrées. Lors des chahuts, par exemple, les garçons peuvent siffler. Or, le sifflet est une technique du corps que la morale bourgeoise réprouve lorsque des jeunes filles la pratiquent. Certes, la domination masculine ne passe pas que par les techniques mais ces dernières jouent un rôle non négligeable dans ce phénomène ; rôle que les travaux de Sigaut permettent d'appréhender, même s'ils n'abordent pas directement la question et supposent donc une lecture peu conventionnelle. L'expérience individuelle et collective du travail, l'attention à la tâche à réaliser et même le plaisir de l'acte technique sont alors des thèmes qui peuvent être inclus dans l'histoire du genre car ils ne sont ni circonscrits à l'histoire sociale ni à celle des techniques.

Sur le plan épistémologique, les rapports entre genre et techniques correspondent à des problématiques "frontières ». Les gestes techniques touchent au corps en même temps qu'aux outils et, plus largement, aux objets. C'est une histoire qui croise celle du travail, travail manuel, donc de l'artisanat, de l'industrie, des mondes paysans et ruraux en même temps qu'une histoire urbaine. C'est une histoire du corps, des gestes du travail et au travail, dans des situations et des espaces variés ${ }^{95}$.

Étudier la production des techniques par le genre et la production du genre par les techniques est encore rare chez les historien·ne.s français.es, mais des travaux récents orientent la réflexion dans ce sens. L'étude de l'histoire des techniques et celle du genre s'en trouvent enrichies. Cependant (re)

91. Guillaume Carnino, L'invention de la science. La nouvelle religion de l'âge industriel, Paris, Seuil, 2015, en particulier, p. 235-257.

92. Émile Durkheim, L'éducation morale, Paris, PUF, rééd. 2012.

93. A. Rauch, Crise de l'identité masculine, op. cit.

94. Michel Foucault, Surveiller et punir, Paris, Gallimard, 1975, p. 209-217.

95. Jacqueline Laufer, Catherine Marry, Margaret Maruani (dir.), Le travail du genre : les sciences sociales du travail à l'épreuve des différences de sexes, Paris, La Découverte, 2003. 
partir des travaux de Mauss nous a permis de montrer que l'histoire des techniques, celle du corps en particulier, sans en faire un sujet de réflexion central ni un objet d'étude particulier, se révèle un champ de recherche riche en outils méthodologiques, encore trop peu utilisés bien qu'existant depuis longtemps, pour comprendre les interactions entre genre et techniques. Plus récemment, le travail de Sigaut a apporté des pistes fécondes. Le corps comme outil ou utilisé comme tel devient un sujet d'étude pour les sciences humaines et sociales qui permet de renouveler les perspectives traditionnelles des rapports aux techniques des deux sexes. Grâce à la connaissance et à la diffusion des travaux de Mauss et de Sigaut, et conjointement à l'évolution économique et sociale du monde occidental, les liens établis entre genre et techniques permettent de rapprocher les apports d'une histoire des techniques, focalisée sur les objets, les systèmes et leurs concepteurs, des avancées historiographiques issues des travaux sur le genre. De cette façon, ces deux auteurs, lus à la lumière des travaux de gender studies produits depuis près d'un demi-siècle, contribuent à un redimensionnement de l'histoire des techniques pouvant inclure pleinement l'histoire du genre (et réciproquement), notamment en dépassant une séparation trop stricte entre l'étude de la conception et celle de l'usage des techniques. De façon à participer au comblement d'un fossé qui a longtemps gêné l'écriture d'une histoire des techniques socialement globalisante: "N'a-t-on pas seulement une histoire d'inventeurs, de techniciens et de concepteurs alors qu'une histoire des usages serait celle des pratiques du grand nombre, une histoire qui d'emblée serait plus féminine, plus orientée vers les oubliés et les dominés, l'histoire de nous tous ?96"

Ces pistes de réflexion sont loin d'être exclusives. Nous l'avons cité plusieurs fois dans cet article, André Leroi-Gourhan a, lui aussi, proposé une anthropologie des techniques et du corps qui, confrontée aux études de genre, peut se révéler féconde pour analyser et comprendre les inégalités entre les sexes induites par les techniques ainsi que la coconstruction du genre et des techniques. C'est un chantier de recherche ouvert.

96. Y. Cohen, D. Pestre, «Présentation », op. cit., p. 734. 


\section{Les auteurs}

Fabien Knittel est maître de conférences en histoire contemporaine à l'Université de Bourgogne Franche-Comté, membre du Centre Lucien Febvre (EA 2273) et chercheur associé aux Archives Henri-Poincaré (UMR 7117, CNRS/Université de Lorraine/Université de Strasbourg). Il est spécialiste d'histoire de l'agronomie et des techniques rurales au xIx siècle.Contact : fabien.knittel@univ-fcomte.fr

Pascal Raggi est maître de conférences HDR à l'Université de Lorraine et chercheur au Centre de recherche universitaire lorrain d'histoire. Ses recherches concernent l'histoire industrielle, minière et sociale au $x x^{\mathrm{e}}$ siècle. En 2013, il a publié, avec Fabien Knittel, Genre et techniques $x x^{e}-\left.x x\right|^{e}$ siècles (Presses universitaires de Rennes, 2013). Il a récemment codirigé avec François Audigier, Les syndicats face à la violence militante des années 1980 à nos jours (Riveneuve, 2018). Il aborde le thème de la désindustrialisation dans ses travaux les plus récents (La désindustrialisation de la Lorraine du fer, Classiques Garnier, 2019). Il est membre de l'Association française pour l'histoire des mondes du travail. Contact : pascal.raggi@univ-lorraine.fr 